\title{
Laparoscopic Extra-peritoneal Para-aortic Node Dissections in the Belfast Trust over the last 9 years: $A$ Single Institution Experience for Locally Advanced Cervical Carcinoma.

\author{
K.Reilly, I.Harley, H.Nagar, E.Craig, S.Dobbs, M.McComiskey \\ Gynae-Oncology Department, Belfast City Hospital, Lisburn Road, BT9 7AB \\ Belfast Health and Social Care Trust, Northern Ireland
}

\section{Objectives:}

Cervical cancer is one of the most commonly occurring female cancers with increasing incidence worldwide. The mainstay of treatment for locally advanced cervical cancer is primary chemoradiotherapy. Pre-operative imaging in combination with laparoscopic extraperitoneal para-aortic node dissection (LEPAND) has been used in order to best target this treatment. This study looked at all women in the Belfast Trust over the last 9 years who had this investigation as part of their pretreatment workup.

\section{Methods:}

Retrospective data was collected for all those who had LEPAND for locally advanced cervical cancer from January 2010 to December 2018. All women had pre-operative imaging that suggested positive pelvic nodes but negative para-aortic nodes.

\section{Results:}

Sixty women were identified in this group ageing 23-69 with median age $39.93 .3 \%$ had stage $2 \mathrm{~b}$ cervical cancer, the remainder were $1 \mathrm{~b} 2$ or $3 \mathrm{~b} .17 \%$ had adenocarcinoma of the cervix and $83 \%$ had squamous cell carcinoma. The median survival overall is 5 years.

$70 \%$ of women had primary LEPAND surgery before chemoradiotherapy with the remainder having primary treatment before surgery.

$15 \%$ of the study population died, $90 \%$ of which died within two years of diagnosis. $67 \%$ of these women had primary LEPAND followed by chemoradiotherapy with the others proceeding straight to primary treatment prior to surgery.

$5 \%(3 / 60)$ had positive para-aortic nodes on histopathology although $100 \%$ appeared node negative on MRI/PET imaging. None with positive nodes had recurrences but $66 \%$ died within 2 years.

\section{Conclusion:}

Despite having negative nodes on imaging 5\% had positive para-aortic lymph nodes on histopathology. LEPAND surgery prevented undertreatment in these women. 